\title{
Utilização de biofertilizantes alternativos no cultivo de palma forrageira: Uma revisão
}

\author{
Use of alternative biofertilizers in the cultivation of forage cactus: A review \\ Uso de biofertilizantes alternativos en el cultivo de palma forrajera: Una revisión
}

Recebido: 12/05/2021 | Revisado: 18/05/2021 | Aceito: 19/05/2021 | Publicado: 06/06/2021

\author{
Orlando Salvador Xavier Junior \\ ORCID: https://orcid.or g/0000-0003-1443-8719 \\ Universidade Federal Rural de Pernambuco, Brasil \\ Unidade Acadêmica de Serra Talhada, Brasil \\ E-mail: orlando.xavierjunior@ufrpe.br \\ Alexandre Maniçoba da Rosa Ferraz Jardim \\ ORCID: https://orcid.org/0000-0001-7094-3635 \\ Universidade Federal Rural de Pernambuco, Brasil \\ E-mail: alexandremrfj@gmail.com \\ Luciana Sandra Bastos de Souza \\ ORCID: https://orcid.org/0000-0001-8870-0295 \\ Universidade Federal Rural de Pernambuco, Brasil \\ Unidade Acadêmica de Serra Talhada, Brasil \\ E-mail: sanddrabastos@yahoo.com.br \\ Carlos André Alves de Souza \\ ORCID: https://orcid.org/0000-0003-1946-0421 \\ Universidade Federal Rural de Pernambuco, Brasi \\ E-mail: carlosandre08_@msn.com \\ José Francisco da Cruz Neto \\ ORCID: https://orcid.org/0000-0002-4889-1282 \\ Universidade Federal Rural de Pernambuco, Brasil \\ E-mail: zenetto.agronomia@gmail.com \\ George do Nascimento Araújo Júnior \\ ORCID: https://orcid.org/0000-0001-9284-4160 \\ Universidade Federal Rural de Pernambuco, Brasil \\ E-mail: georgearaujo.agro@gmail.com \\ Renan Matheus Cordeiro Leite \\ ORCID: https://orcid.org/0000-0001-8454-8660 \\ Universidade Federal Rural de Pernambuco, Brasil \\ Unidade Acadêmica de Serra Talhada, Brasil \\ E-mail: renanmatheuscl@gmail.com \\ Thieres George Freire da Silva \\ ORCID: https://orcid.org/0000-0002-8355-4935 \\ Universidade Federal Rural de Pernambuco, Brasil \\ Unidade Acadêmica de Serra Talhada, Brasil \\ E-mail: thigeoprofissional@hotmail.com.br
}

\begin{abstract}
Resumo
A palma (Opuntia e Nopalea) é uma das forrageiras mais importantes do mundo, nativa do México, pertencendo à família Cactaceae, e possui uma alta eficiência no uso da água. No Brasil é cultivada principalmente no Nordeste, onde é considerada estratégica devido as condições climáticas da região. Pesquisas indicam que a adubação orgânica induz um melhor desempenho produtivo da palma forrageira. Neste sentido, o uso de biofertilizante para suprir as necessidades nutricionais das culturas agrícolas, tem sido uma importante ferramenta para os produtores rurais, por se tratar de um insumo orgânico de baixo custo, resultante da fermentação aeróbica de matéria orgânica e água, e que pode influenciar positivamente as propriedades químicas e físicas do solo. Esta revisão de literatura tem por objetivo apresentar alternativas de práticas agrícolas com adubação orgânica como o uso de biofertilizantes em sistemas de cultivo com palma forrageira visando alternativas promissoras com eficiência de cultivo. Conclui-se que o cultivo de palma forrageira com utilização de biofertilizantes alternativos orgânicos, incrementam a produção das plantas, e viabilizar técnicas de baixo custo aos produtores rurais.
\end{abstract}

Palavras-chave: Adubação; Opuntia stricta; Nopalea cochenillifera; Solo.

\section{Abstract}

The forage cactus (Opuntia and Nopalea) is one of the most important forages in the world, native to Mexico, belonging to the Cactaceae family, and has high water-use efficiency. In Brazil, it is grown mainly in the Northeast, 
where it is considered strategic due to the climatic conditions of the region. Research indicates that organic fertilization induces a better productive performance of the forage cactus. In this sense, the use of biofertilizer to supply the nutritional needs of agricultural crops has been an important tool for rural producers, as it is a low-cost organic input, resulting from the aerobic fermentation of organic matter and water, and which can positively influence the chemical and physical properties of the soil. This literature review aims to present alternatives of agricultural practices with organic fertilization such as the use of biofertilizers in cultivation systems with forage cactus aiming at promising alternatives with efficient cultivation. It is concluded that the cultivation of forage cactus with the use of alternative organic biofertilizers, increases the production of plants, and enables low-cost techniques to rural producers.

Keywords: Fertilizing; Opuntia stricta; Nopalea cochenillifera; Soil.

\begin{abstract}
Resumen
El cactus forrajero (Opuntia y Nopalea) es uno de los forrajes más importantes del mundo, originario de México, perteneciente a la familia Cactaceae, y tiene una alta eficiencia en el uso del agua. En Brasil se cultiva principalmente en el Nordeste, donde se considera estratégico debido a las condiciones climáticas de la región. Las investigaciones indican que la fertilización orgánica induce un mejor desempeño productivo del nopal forrajero. En este sentido, el uso de biofertilizantes para suplir las necesidades nutricionales de los cultivos agrícolas ha sido una herramienta importante para los productores rurales, por ser un insumo orgánico de bajo costo, resultado de la fermentación aeróbica de materia orgánica y agua, y que puede positivamente influyen en las propiedades químicas y físicas del suelo. Esta revisión de la literatura tiene como objetivo presentar alternativas de prácticas agrícolas con fertilización orgánica como el uso de biofertilizantes en sistemas de cultivo con cactus forrajero con el objetivo de alternativas prometedoras con cultivo eficiente. Se concluye que el cultivo de cactus forrajero con el uso de biofertilizantes orgánicos alternativos, aumenta la producción de plantas y habilita técnicas de bajo costo para los productores rurales.
\end{abstract}

Palabras clave: Fertilizante; Opuntia stricta; Nopalea cochenillifera; Suelo.

\title{
1. Introdução
}

A agricultura convencional, mesmo com diversas vantagens econômicas, proporciona significativos impactos ambientais negativos que não são incorporados aos custos de produção dos sistemas agrícolas. Esta atividade utiliza adubos minerais que contribuem para a poluição do solo e dos corpos hídricos. Outro problema da utilização deste tipo de fertilizante industrializado é o exaurimento de reservas de minerais e petróleo, o qual inviabiliza a sustentabilidade dos recursos naturais, tendo em vista que estes recursos têm reserva finita (Lana, 2009; Lassaletta et al., 2014).

Nas últimas décadas houve um crescimento demasiado na utilização de fertilizantes químicos (Liu et al., 2020). Segundo a Associação Nacional para Difusão de Adubos (ANDA) em 2011, o consumo de fertilizantes minerais ultrapassou 29 milhões de toneladas, destes mais de $60 \%$ foram importados. Neste sentido, a adubação orgânica é uma alternativa aos fertilizantes minerais que proporcionam diversos benéficos as características do solo (físicas e químicas), e são oriundos de recursos finitos e de elevado custo para aquisição (Schumacher et al., 2001; Paungfoo-Lonhienne et al., 2019; Silva et al., 2020a).

A utilização de resíduos orgânicos oriundos da criação de animais e subprodutos agrícolas é uma alternativa antiga e promissora para a fertilização dos cultivos (Komiyama et al., 2013; Paolini et al., 2018). A substituição de insumos industriais por recursos disponíveis na natureza e propriedades rurais, é o primeiro passo no processo de transição agroecológica, entretanto, ainda é escasso o conhecimento sobre as quantidades e dinâmica no solo destes produtos que devem ser aplicados para obtenção de rendimentos satisfatórios na produção das culturas agrícolas (Figueroa et al., 2012; Rothé et al., 2019; Amoah-Antwi et al., 2020) (Figura 1). 
Figura 1. Utilização de resíduos orgânicos para melhoria do solo no setor agrícola. Fonte.

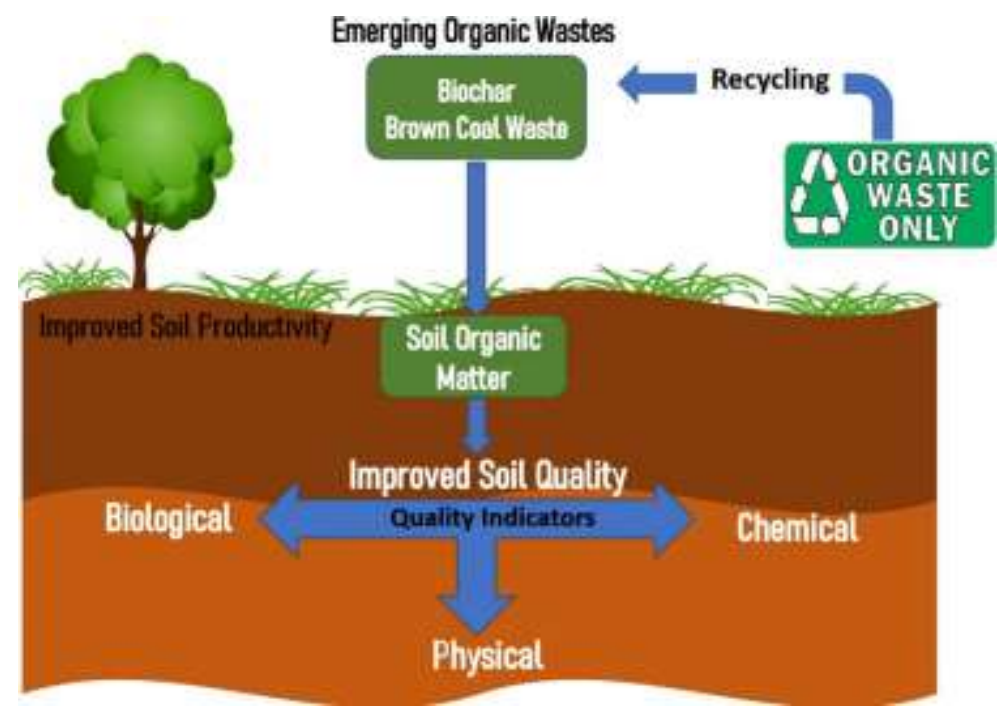

Fonte: Amoah-Antwi et al. (2020).

Como cultura de expressiva importância econômica e rusticidade nos sistemas de cultivo de ambientes semiáridos, a palma forrageira (Opuntia spp. e Nopalea spp.) além de ser uma planta muito adaptada as condições hostis de clima e solo, possui respostas positivas no incremente de práticas de adubação com fertilizantes industriais (Silva et al., 2014; Silva et al., 2020b; Jardim et al., 2020a; Jardim et al., 2021a), e respostas promissoras com o uso de adubos orgânicos, como o esterco animal (Dubeux Júnior \& Santos, 2005; Santos et al., 2006). Contudo, existe lacunas na literatura na utilização de biofertilizantes formulados com resíduos agrícolas orgânicos somado ao esterco animal do setor agropecuário.

Assim, objetivou-se com esta revisão apresentar alternativas de práticas agrícolas com adubação orgânica como o uso de biofertilizantes em sistemas de cultivo com palma forrageira visando alternativas promissoras com eficiência de cultivo.

\section{Metodologia}

A presente revisão sistemática fundamentou-se em trabalhos científicos indexados em bases de dados eletrônicas como ScienceDirect, Scopus e Google Scholar sem restrição de idiomas, baseando-se em materiais com significativa relevância científica em ciências agrárias e ambientais, contribuindo para melhor entendimento do assunto (Souza et al., 2009; Pereira et al., 2018). Vale ressaltar que devido ao amplo uso de produtos como fertilizantes agrícolas o trabalho não se limitou a estudos da última década.

Como estratégia de busca do material científico para compor a revisão, foram utilizados termos de indexação e palavras-chave como: agriculture, fertilizers, soil, tratos culturais, palma forrageira, Opuntia, Nopalea, crop, sustentabilidade, management, organic manure, feedstock type, waste, environmental pollution, and farms.

\section{Revisão de Literatura}

\subsection{Uso de biofertilizantes na agricultura}

O declínio na qualidade de vida rural e a degradação dos recursos naturais associados à agricultura pós Revolução Verde, impulsionou a discussão sobre o desenvolvimento sustentável neste meio, tornando a produção agrícola não apenas uma questão técnica, mas um processo condicionado por dimensões ambientais, sociais, culturais, políticas e econômicas, que devem ser compatibilizadas em agroecossistemas sustentáveis (Altieri, 2009; Urra et al., 2020). A sustentabilidade de um 
sistema agrícola requer o uso racional dos recursos naturais, tendo em vista a perpetuidade produtiva deste bem. O modelo convencional de agricultura requer um aporte elevado e contínuo de insumos industriais para a manutenção do sistema, apresentando alto custo na compra do material e causando impactos aos ecossistemas (Gliessman, 2000; Urra et al., 2020; Hrustek, 2020) (Figura 2).

Figura 2. Pilares da agricultura sustentável.

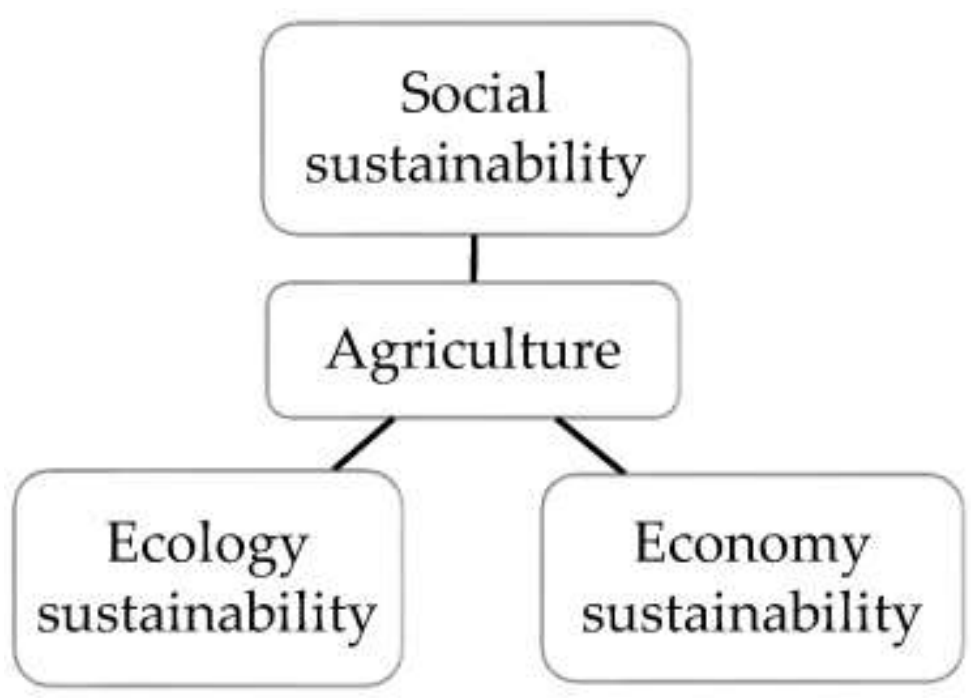

Fonte: Hrustek (2020).

A diminuição do consumo das reservas naturais, bem como o aumento dos custos de produção, tem despertado o interesse de estudiosos direcionando-os há diminuição do uso dos fertilizantes minerais (Villela Júnior et al., 2003; Riva et al., 2016; Urra et al., 2020). Neste contexto, a utilização de materiais orgânicos como fonte de matéria-prima alternativa para produção de fertilizantes é uma medida estratégica e valiosa do ponto de vista ambiental, sendo conveniente, desde que seja viável, também, do ponto de vista econômico (Fernandes et al., 2003; Chojnacka et al., 2019).

O uso de adubos orgânicos, além de melhorar a agregação do solo, aeração, temperatura e penetração de raízes, também é capaz de integrar ao solo compostos orgânicos que são decompostos e transformados em nutrientes essenciais disponíveis às plantas (Oliveira et al., 2009; Paolini et al., 2018; Jardim et al., 2018). A adição de matéria orgânica no solo provoca a estabilidade dos agregados, melhorando a infiltração, percolação e retenção de água, além de aumentar a capacidade de troca catiônica (CTC) e o suprimento dos nutrientes (Du Preez et al., 2011; Jardim et al., 2017; Alves et al., 2018a). Os fertilizantes orgânicos atuam no solo enriquecendo-o gradualmente com macro e micronutrientes essenciais às plantas (Trani et al., 2013). Para Malavolta et al. (2002), como exemplo dos adubos orgânicos, destacam-se os biofertilizantes por proporcionar bons resultados na agricultura e com diversas formas de aplicação, dosagens e concentrações.

Os biofertilizantes são definidos conforme a Instrução Normativa nº 46 de 06 de outubro de 2011, como produtos que contêm componentes ativos ou agentes biológicos capazes de atuar, direto ou indiretamente, sobre o todo ou sobre partes das plantas cultivadas, melhorando o desempenho do sistema de produção, e, que sejam isentos de substâncias proibidas pela regulamentação de orgânicos (MAPA, 2012). O uso desta modalidade de fertilizante vem crescendo em diversos países do mundo, devido à busca crescente de produtos menos agressivos ao meio ambiente, bem como ser de fácil alcance e produção, além de incrementar na produtividade das culturas (Medeiros et al., 2007; Kour et al., 2020). O uso desses produtos pode servir de suporte para uma agricultura sustentável e economicamente viável para os pequenos produtores (Boraste et al., 2009). O emprego dos biofertilizantes influencia diretamente na fertilidade do solo, fornecendo nutrientes, resultantes da ação 
metabólica dos microrganismos benéficos que formam compostos quelatizados, sendo disponibilizados e prontamente absorvidos pelas plantas (Araújo et al., 2007; Owamah et al., 2014; Kour et al., 2020).

Em comparação com os fertilizantes minerais, os biofertilizantes apresentam inúmeros benefícios, como tornar o solo mais poroso, permitindo maior aeração em camadas mais profundas, estabelecimento do equilíbrio nutricional, preservação das interações biológicas, tornando o solo favorável a multiplicação de microrganismos benéficos, proporcionando maior eficiência nutricional e fotossintética; e consequentemente maior produtividade e qualidade dos cultivos (Izumi et al., 2010; Moraes, 2015; Chojnacka et al., 2019; Mitter et al., 2021) (Figura 3). Assim, o uso desse tipo de produto por pequenos produtores é uma alternativa viável e econômica, como uma prática recomendada não somente para fins de fertilização, mas também no controle fitossanitário, reduzindo os custos com insumos e defensivos (Primavesi et al., 2004).

Figura 3. Interação dos nutrientes no solo e micorganismos no sistema solo-planta.

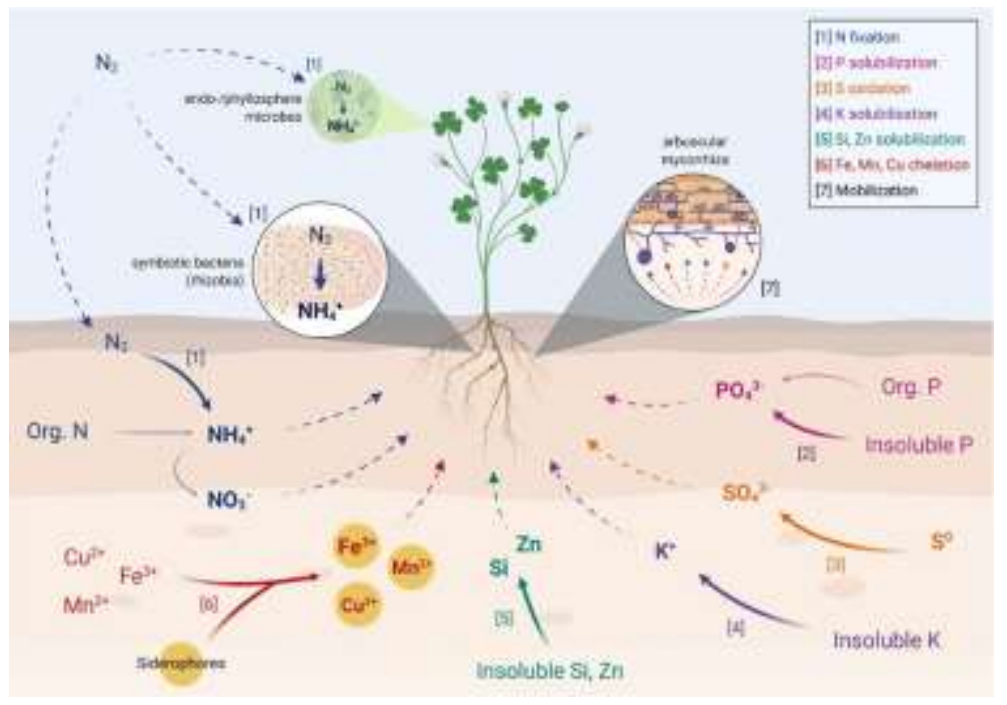

Fonte: Mitter et al. (2021).

A utilização de biofertilizantes na nutrição de culturas agrícolas, é uma prática que pode favorecer tanto ao meio ambiente quanto aos produtores, pois este contém os nutrientes essenciais para o crescimento das plantas, proporciona a melhoria das características químicas, físicas e biológicas do solo, além de reduzir o uso de agroquímicos e os custos com o cultivo, o que eleva a sustentabilidade dos sistemas agrícolas (Souza et al., 2010; Souza et al., 2018). Segundo Hamoudi \& Larich (2013), os insumos orgânicos em geral, entre eles os biofertilizantes, aumentam os espaços porosos para a infiltração e retenção da água, isto possibilita em algumas situações, maior crescimento radicular, exercendo efeitos benéficos sobre o crescimento das plantas, trocas gasosas e extração de nutrientes, sendo que as doses a serem empregadas dependerão do tipo, textura, estrutura e teor de matéria orgânica presente no solo (Silva et al., 2013; Kour et al., 2020).

A absorção pelas plantas se efetua com muita rapidez, de modo que é muito útil para as culturas de ciclo curto, ou no tratamento rápido de deficiências nutricionais das plantas (Lovatto et al., 2011). Maghanaki et al. (2013) reportaram que os biofertilizantes líquidos são absorvidos com maior facilidade pelo solo do que os fertilizantes sólidos, já que os mesmos entram em contato direto com as raízes da planta, sendo que o sólido necessita de água da chuva e/ou irrigação para dissolver-se e torna-se disponível para as plantas. A importância do biofertilizante no crescimento das plantas não se deve aos valores quantitativos dos seus componentes químicos que, em geral, são baixos, mas no aspecto qualitativo, devido sua diversidade química (Borges et al., 2012); visto que, sua utilização não gera problemas ao solo, como acidez e degradação do mesmo, pelo fato de ter um pH levemente alcalino (Barbosa \& Langer, 2011). 
O preparo de caldas a base de biofertilizantes tem se difundido como um método de reciclagem de resíduos orgânicos industriais, e estercos para uso no manejo de plantas (Paré et al., 1998). Dessa forma, minimiza-se também a poluição ambiental e a degradação do solo, reduz-se o descarte de resíduos e limita-se a emissão de gases de efeito estufa. Os biofertilizantes são compostos bioativos, resíduos finais da fermentação de compostos orgânicos, que contêm células vivas ou latentes de microrganismos (por exemplo, bactérias, leveduras, bacilos, algas e fungos filamentosos) e seus metabólitos, além de quelatos organo-minerais (Alves et al., 2001; Kour et al., 2020). Geralmente, o ingrediente mais utilizado para a produção do biofertilizante é o esterco de animais, principalmente por conter na sua composição macro e micronutrientes essenciais as plantas, além da sua grande disponibilidade na maioria dos estabelecimentos agrícolas (Probst et al., 2009).

A produção de biofertilizante é decorrente do processo de fermentação (e.g., aeróbica ou anaeróbica), ou seja, da atividade dos microrganismos na decomposição da matéria orgânica e complexação de nutrientes, o que pode ser obtido com a simples mistura de água e esterco fresco (Lovatto et al., 2011; Urra et al., 2020). Podendo possuir composição altamente complexa e variável, dependendo do material empregado, contendo a maioria dos nutrientes necessários aos vegetais (Melo et al., 2009). Meirelles et al. (1997), explicam que os biofertilizantes tem um tempo de fermentação concluída em 30 dias no verão, ou 45 dias no inverno. A temperatura é um dos fatores importantes para sua fermentação, sendo que no período quente pode ser concluída de 14 a 30 dias e em períodos mais frios, de 45 a 90 dias, sendo que a temperatura ideal é $38{ }^{\circ} \mathrm{C}$. Uma vantagem no uso do biofertilizante, é que pode ser produzido pelo próprio agricultor, gerando economia de insumos industrializados (Medeiros et al., 2008).

A biodigestão aeróbica consiste em um processo biológico que acontece na presença de oxigênio, no qual diversas populações de bactérias convertem a matéria orgânica em uma mistura de metano, dióxido de carbono e pequenas quantidades de hidrogênio, nitrogênio e sulfitos (Parawira, 2004; Chojnacka et al., 2019). De acordo com D’Andrea \& Medeiros (2002), o processo de fermentação é complexo e os microrganismos existentes no produto, passam quatro fases distintas de crescimento celular:

- Latência: compreende o período de adaptação dos microrganismos, após o qual as células dão início à fermentação;

- Crescimento exponencial: nessa fase ocorre elevado processo de biomassa e liberação dos metabólitos primários: carboidratos, aminoácidos, lipídeos, nucleotídeos, vitaminas, proteínas e enzimas;

- Fase estacionária: as células param de se dividir e as colônias, após juntarem-se, iniciam um processo de diferenciação celular produzindo metabólitos secundários como forma de defesa (antibióticos, toxinas, fenóis, ácidos orgânicos e outras proteínas de cadeia longa, de alto interesse biotecnológico); e,

- Morte celular: esgotadas as reservas de energia, as células começam a morrer numa velocidade exponencial.

Os biofertilizantes diferem dos fertilizantes minerais convencionais podem ser produzidos em diversos lugar e diferentes matérias prima, inclusive resíduos de sistemas agropecuários. Segundo Restrepo Rivera (2014), os principais ingredientes utilizados no preparo dos biofertilizantes segue abaixo e suas respectivas funções:

- Leite ou soro de leite: são ricos em aminoácidos, vitaminas, lipídios e proteínas, ingredientes necessários para proporcionar as condições adequadas para os microrganismos se multiplicarem. Tem a importância na formação de outros compostos orgânicos que são estimulados durante o processo.

- Melaço de cana-de-açúcar: é responsável pela energia necessária para que os microrganismos realizem o trabalho de decomposição da matéria. O melaço dissolvido em água impulsiona o processo de fermentação, isto é, auxilia o desenvolvimento de microrganismos encarregados de transformar a matéria orgânica em nutrientes fáceis de assimilar pelas plantas. É rico em potássio, cálcio, fósforo, magnésio e micronutrientes 
como boro, zinco e ferro.

- Cinza vegetal: contém grande aporte de microelementos, favorece as características físicas do solo, permitindo uma melhor distribuição das raízes, uma maior aeração e absorção de umidade e fluxo de energia. Seu alto grau de porosidade incrementa a oxigenação do composto beneficiando a atividade microbiana, pois retêm, filtra e libera nutrientes, atuando como regulador da temperatura do solo aumentando a resistência das raízes, e liberando gradualmente nutrientes úteis as plantas diminuindo a perda e lixiviação destes no solo.

- Pó de rocha: importante fonte de sais minerais que além de enriquecer e ativar a fermentação, nutre e fertiliza o solo e as plantas.

- Suco de frutas: é um material benéfico para microfauna em estágios diferentes de decomposição, e desta forma, rico em uma microbiota diversificada. É um ingrediente rico em vitaminas complexas que estimulam a ativação de hormônios de crescimento, aumentando a fermentação do composto.

- Esterco animal: é a principal fonte de microrganismos que estimulam a fermentação, grande inóculo: leveduras, fungos, bactérias, protozoários sendo responsáveis fermentativo. Sua grande vantagem é que permitir tanto a fermentação aeróbica como anaeróbica. Material rico em nitrogênio, melhora a qualidade e a fertilidade do solo com nutrientes como fósforo $(\mathrm{P})$, potássio $(\mathrm{K})$ cálcio $(\mathrm{Ca})$, magnésio $(\mathrm{Mg})$, zinco $(\mathrm{Zn})$, cobre $(\mathrm{Cu})$ e boro $(\mathrm{B})$. Em geral, o esterco melhora as condições biológicas, químicas e físicas dos solos. $\mathrm{O}$ esterco fresco apresenta uma gama de microrganismos vivos que são indispensáveis para o início do processo de fermentação do biopreparado.

Desta forma, a eficiência deste produto depende de características da matéria orgânica biodigerida do manejo do produto (época, dosagens e forma de aplicação), das características edafoclimáticas e do conhecimento dos mecanismos e interações entre os microrganismos e a fração mineral do solo (Abdel Monem et al., 2001), para que os mesmos sejam utilizados nas culturas. Assim, a escolha do tipo de cultura em uma área agrícola é de suma importância, para aplicação eficiente de biofertilizantes e o entendimento de suas respostas a aplicação.

\subsection{Origem e distribuição da palma forrageira}

A palma forrageira (Opuntia e Nopalea) é uma das plantas mais eficientes no armazenamento de água e biomassa, originária do México pertencente à família Cactaceae. É uma espécie com forte expressão econômica e social, caracterizandose como uma das plantas forrageiras mais importantes do mundo, atualmente está presente em todos os continentes, sendo utilizada para diversos fins e sistemas de cultivo, destacando-se o uso na alimentação animal (Silva et al., 2014; Meza-Herrera et al., 2019; Jardim et al., 2019; Jardim et al., 2020b). Foi introduzia no Brasil por volta de 1880, pelo estado de Pernambuco, por meio de cladódios provenientes do Texas, EUA (Nunes, 2011). A maior área cultivada com palma forrageira no mundo, está na região Nordeste do Brasil, alcançando cerca de 550 mil hectares, sendo a área plantada do Brasil de aproximadamente 600.000 ha (Araújo et al., 2005; Dubeux Júnior et al., 2013; Garcete-Gómez et al., 2017).

Entre as diversas forrageiras que existem no semiárido brasileiro, como gramíneas e plantas nativas, esta cactácea destaca-se em virtude dos seus mecanismos de adaptações à escassez de água, o que permite a sua perenidade em ambientes de extrema condição de aridez, mantendo a sustentabilidade da pecuária no semiárido, proporcionando produção quantitativa e qualitativa, através da melhoria na dieta alimentar a custos razoáveis para os produtores rurais (Alencar et al., 2011; Barbosa et al., 2017; Jardim et al., 2020b; Jardim et al., 2021b; Silva et al., 2020c). A palma forrageira possibilita a produção animal nos períodos mais críticos do ano, surgindo como fonte alternativa de alimento, pois oferece boa disponibilidade no período seco, bom coeficiente de digestibilidade da matéria seca e alta produtividade. Além de fornecer um alimento verde, supre grande 
parte das necessidades de água dos animais na época de escassez, desse modo, pode ser introduzida na alimentação de bovinos, caprinos e ovinos (Santos et al., 1997; Alves et al., 2018b; Nunes et al., 2019; Silva et al., 2021a).

Devido à sazonalidade da vegetação nas regiões áridas e semiáridas esta cultura é uma alternativa promissora para suprir a alimentação animal, tornando-a assim opção de cultura xerófila com maior potencial de exploração na pecuária do Nordeste brasileiro (Cândido et al., 2013), fator esse devido à alta eficiência produtiva e, exigência mínimas de água (Lima et al., 2018; Araújo Júnior et al., 2021).

\subsubsection{Características botânicas e morfológicas}

Uma das razões da ampla distribuição desta cactácea nos continentes é decorrente da sua adaptação anatômica, bioquímicas, morfológicas e fisiológica as regiões áridas e semiáridas. A palma forrageira possui uma alta eficiência no uso da água devido alguns aspectos fisiológicos, como a cutícula impermeável, menor número de estômatos e eficaz aparelho fotossintético (Ramos et al., 2011; Lima et al., 2018). O grande diferencial da mesma é seu metabolismo CAM (crassulasean acid metabolism), considerando a chave para o sucesso e sobrevivência em regiões áridas e semiáridas. As plantas CAM mantêm seus estômatos fechados durante o dia e abrem durante a noite, quando geralmente as temperaturas são mais baixas, minimizando a perda de água, pois a $\mathrm{H}_{2} \mathrm{O}$ e $\mathrm{CO}_{2}$ têm a mesma via de difusão (Taiz \& Zeiger, 2004; Borland et al., 2014; Jardim et al., 2021b).

Em todo mundo, já foram descritas cerca de 2.000 espécies e 178 gêneros de palma forrageira, sendo os gêneros Opuntia e Nopalea com expressiva utilização como fonte de forragem. No semiárido nordestino, principalmente no estado de Pernambuco, são cultivadas as espécies $O$. stricta (Haw.) Haw., do clone Orelha de Elefante Mexicana, e a N. cochenillifera (L.) Salm-Dyck, representadas pelos clones IPA Sertânia e Miúda. No entanto, a décadas passadas, os palmais eram muito difundidos com os clones Gigante, Redonda e IPA-20, porém, devido a suscetibilidade a praga principal da cultura, a cochonilha-do-carmim (Dactylopius opuntiae Cockerell, 1929; Hemiptera: Dactylopiidae), houve uma drástica diminuição de cultivos com o uso de clones não resistes a praga (Santos et al., 2006; Cavalcanti et al., 2008; Jardim et al., 2020b; Silva et al., 2021b).

Os clones de Opuntia apresenta tolerância à cochonilha-do-carmim (D. opuntiae), cladódios grandes e suculentos, flores na maioria hermafroditas, presença de espinhos, e uma menor exigência em fertilidade do solo. Em relação a Nopalea, tem porte pequeno e caule ramificado e mais achatados, apresentando maiores teores de matéria seca e carboidrato; sendo assim, mais nutritiva, podendo ter clones inermes e outros com aspecto espinhoso. É mais exigente em fertilidade e umidade, e tem menor tolerância à seca, embora seja resistente à cochonilha-do-carmim (Santos et al., 2006; Cavalcanti et al., 2008; Vasconcelos et al., 2009; Jardim et al., 2020b).

\subsubsection{Exigências edafoclimáticas e nutricionais}

Um fator preponderante da palma forrageira é a sua rusticidade, que proporciona desenvolvimento satisfatório em região com baixos índices pluviométricos. Entretanto, informações sobre umidade relativa do ar e do solo, temperatura do ar média do dia e da noite são determinantes na produção desta cactácea. De acordo com zoneamento elaborado por Souza et al. (2008), à máxima capacidade produtiva da cultura, ocorre em regiões com temperatura média varia entre $16,1^{\circ} \mathrm{C}$ e $25,4^{\circ} \mathrm{C}$; com máximas entre $28,5^{\circ} \mathrm{C}$ e $31,5^{\circ} \mathrm{C}$ e, temperatura mínimas variando de 8,6 a $20,4{ }^{\circ} \mathrm{C}$. A faixa ideal de chuva concentra-se entre $368,4 \mathrm{~mm}$ e $812,4 \mathrm{~mm}$; podendo também ser cultivada com mínimos volumes de chuva (200 $\mathrm{mm}$ ), e a umidade relativa

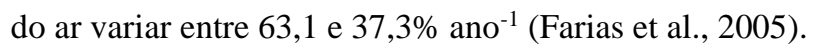

Os cultivos de palma forrageira podem ser indicados em áreas de solo com textura do tipo arenosa à argilosa, mas, frequentemente recomenda-se o plantio em solos argilo-arenosos. $\mathrm{O}$ pH do solo pode variar de 5,5 a 7,5, demonstrando boa 
adaptação da cultura; além do mais, é de fundamental importância também, a boa drenagem, uma vez que áreas sujeitas a encharcamento não se adequam ao cultivo da palma forrageira, ocasionado problemas ao desenvolvimento da planta e até morte do estande (Santos et al., 2006; Jardim et al., 2021a). Nos solos da região semiárida brasileira, quando não há um manejo adequado de adubação, a produtividade da palma forrageira é comprometida ao longo do tempo devido à redução da fertilidade (Dubeux Júnior \& Santos, 2005), decorrente da exigência principalmente em Ca e Mg no solo (Sampaio, 2005).

$\mathrm{Na}$ busca da manutenção de elevados rendimentos de fitomassa, há necessita de tratos culturais como adubação, controle de plantas espontâneas e espaçamento adequado de cultivo, podendo a produção de matéria seca variar de 12 a 47 toneladas (Nascimento et al., 2002). Quando se prioriza a adubação orgânica, pode-se empregar esterco bovino, ovino ou caprino, na dose 10 a $30 \mathrm{t} \mathrm{ha}^{-1}$ em fundação e em cobertura a cada dois anos, no período que antecede as primeiras chuvas (Santos et al., 2006). Resultados obtidos em estudos realizados com palma forrageira utilizando esterco animal, incrementou a produtividade desta cultura, sendo estes achados superiores aos obtidos com o uso isolado de fertilizantes químicos (Dubeux Júnior \& Santos, 2005).

\subsubsection{Importância socioeconômica}

Em regiões áridas e semiáridas, como na do Brasil, a palma forrageira possui nítida importância na alimentação dos animais, visando uma estratégia de convivência com a seca. Os principais fatores que determinam o incentivo do cultivo desta cactácea, é a ofertando alternativas de alimentos (cladódios e frutos) com alta qualidade nutricional para os animais; a renda com as atividades pecuárias (e.g. venda dos cladódios), que auxiliaram na garantia da segurança alimentar da população; e sua rusticidade que promove adaptações as condições climáticas adversas previstas para as próximas décadas (Embrapa, 2012; Alves et al., 2019).

A contribuição positiva da viabilidade econômica da cultura nas pequenas e médias propriedades, notadamente na alimentação dos rebanhos (Galindo et al., 2005; Carvalho et al., 2017), proporciona incentivo do seu cultivo, sendo considerada uma das opções mais promissoras para o rendimento de forragem em ambientes hostis, podendo atingir altas produtividades, e diminuir a sazonalidade da disponibilidade de forragem ao longo do ano (Menezes et al., 2005). No Brasil, apesar deste cacto apresentar uso em maiores volumes para a produção de biomassa, vale salientar que o gênero Opuntia, além do alto rendimento de forragem, produz frutos de qualidade com características nutracêuticas e farmacológicas.

\subsubsection{Uso da matéria orgânica na adubação da palma forrageira}

A utilização de esterco no semiárido nordestino na cultura da palma forrageira é bastante restrita, apesar da criação de ruminantes ser uma das principais atividades econômicas, o homem do campo diante das suas necessidades e falta de conhecimento da importância desse material para as plantas, e também por tradição, vendem o esterco produzido pelos animais, para incrementar a renda da família. Técnicas que visam a adição de fertilizantes orgânicos podem promover para uma maior conscientização da importância na matéria orgânica nos cultivos agrícolas (Melo et al., 2009; Attwood et al., 2019) (Figura 4). 
Figura 4. Esterco animal como reposição de matéria orgânica em pastagem.

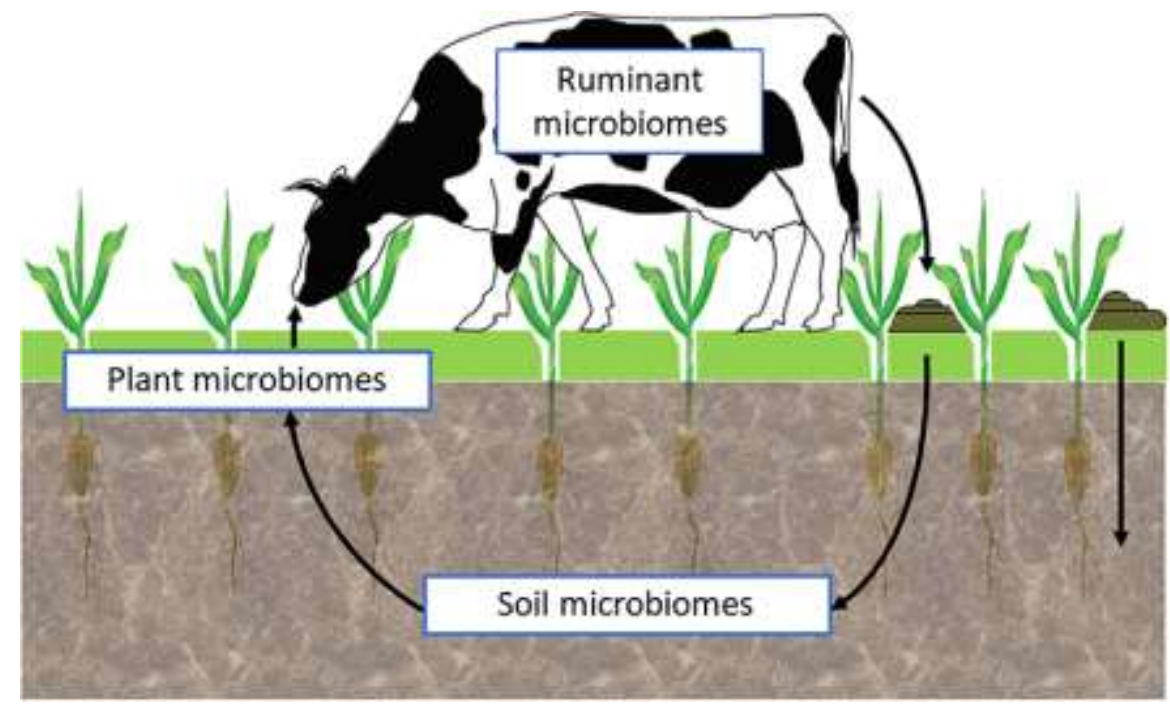

Fonte: Attwood et al. (2019).

A palma responde bem a adubação orgânica, e como os solos do semiárido, em sua maioria, apresentam baixo teor de matéria orgânica, a prática da fertilização é uma necessidade nos cultivos (Sales et al., 2014). No caso de se optar pela adubação orgânica, pode ser utilizado esterco bovino ou caprino, na quantidade de 10 a $30 \mathrm{t}^{-1} \mathrm{a}^{-1}$ no plantio e a cada dois anos, no período próximo à estação chuvosa. Dubeux Júnior \& Santos (2005) recomendaram o uso de $10 \mathrm{a} 30 \mathrm{t}$ ha $^{-1} \mathrm{de}$ esterco bovino após cada colheita, dependendo do espaçamento de plantio utilizado. Santos et al. (2008) observaram resposta linear da produtividade do clone IPA-20 até $80 \mathrm{t} \mathrm{ha}^{-1}$ de esterco bovino aplicado.

A adubação com biofertilizante caprino aumenta os teores de bases no solo quando comparada a adubação mineral, e, pode substituir de forma viável a adubação mineral (Marques et al., 2015). Nos sistemas agrícolas do semiárido, são fundamentais os manejos e a conservação do solo para a manutenção adequada das características físicas, químicas e biológicas. Assim, para obtenção de êxito nos cultivos, solo deve apresentar quantidade equilibrada de nutrientes, matéria orgânica e microrganismos (Borges \& Bettiol, 2010; Biesdorf et al., 2019; Jardim et al., 2020c).

\section{Considerações Finais}

As informações apresentadas nessa revisão esclarecem estratégias de cultivos de palma forrageira como a utilização de biofertilizantes alternativos orgânicos, com intuito de incrementar a produção das plantas, e viabilizar técnicas de baixo custo aos produtores rurais. Além disso, por se tratar de uma planta forrageira, a manutenção de suas exigências nutricionais poderá favorecer no incremento de rendimento, tornando-se a oferta de forragem menos sazonais durante o ano, e principalmente em períodos de estiagem.

Contudo, apesar de ser uma cultura de expressivo uso no setor agropecuário, estudos futuros devem ser realizados, e mais pesquisas de campo são necessárias para entender os principais constituintes utilizados nos biofertilizantes, quais às doses e época do ano para aplicação desse material na palma forrageira, visando maiores rendimentos e qualidade na produção de forragem.

\section{Agradecimentos}

Os autores gostariam de agradecer o apoio financeiro da Coordenação de Aperfeiçoamento de Pessoal de Nível 
Superior (CAPES - Código de Financiamento 001), Fundação de Amparo à Ciência e Tecnologia do Estado de Pernambuco (FACEPE - APQ-0215-5.01/10 e FACEPE - APQ-1159-1.07/14), e ao Conselho Nacional de Desenvolvimento Científico e Tecnológico (CNPq - 475279/2010-7, 476372/2012-7, 305286/2015-3, 309421/2018-7, e 152251/2018-9) pelo apoio financeiro e concessão das bolsas dos autores.

\section{Referências}

Abdel Monem, M. A. S., Khalifa, H. E., Beider, M., Ghandour, I. A. E., \& Galal, Y. G. (2001). Using biofertilizers for maize production: response and economic return under different irrigation treatments. Journal of Sustainable Agriculture, 19(2), 41-48. https://doi.org/10.1300/J064v19n02_05

Alencar, L. P. D., Sediyama, G. C., Wanderley, H. S., Almeida, T. S., \& Delgado, R. C. (2011). Avaliação de métodos de estimativa da evapotranspiração de referência para três localidades no norte de Minas Gerais. Engenharia na Agricultura, 19(5), 437-449.

Altieri, M. (2009). Agroecologia: a dinâmica produtiva da agricultura sustentável. 5. ed. Porto Alegre. Editora da UFRGS. 117 p.

Alves, H. K. M. N., Jardim, A. M. R. F., Souza, L. S. B., \& Silva, T. G. F. (2018a). The application of agrometeorological techniques contributes to the agricultural resilience of forage cactus: A review. Amazonian Journal of Plant Research, 2, 207-220. https://doi.org/10.26545/ajpr.2018.b00026x

Alves, H. K. M. N., Jardim, A. M. R. F., Alves, C. P., Souza, L. F., Fonsêca, G. R. F., Silva, A. R., Santos, T. S., \& Silva, T. G. F. (2018b). Acúmulo de água na palma forrageira em decorrência de práticas de resiliência no Semiárido pernambucano.

Alves, S. B., Medeiros, M. D., Tamai, M. A., \& Lopes, R. B. (2001). Trofobiose e microrganismos na proteção de plantas: biofertilizantes e entomopatógenos na citricultura orgânica. Biotecnologia Ciência \& Desenvolvimento, 21(16), 16-21.

Alves, C. P., Silva, T. G. F., Alves, H. K. M. N., Jardim, A. M. R. F., Souza, L. S. B., Cruz Neto, J. F., \& Santos, J. P. A. S. (2019). Consórcio palma-sorgo sob lâminas de irrigação: balanço de água no solo e coeficientes da cultura. Agrometeoros, 27(2). http://dx.doi.org/10.31062/agrom.v27i2.26448

Amoah-Antwi, C., Kwiatkowska-Malina, J., Thornton, S. F., Fenton, O., Malina, G., \& Szara, E. (2020). Restoration of soil quality using biochar and brown coal waste: A review. Science of the Total Environment, 722, 137852. https://doi.org/10.1016/j.scitotenv.2020.137852

ANDA - Associação Nacional para Difusão de Adubos. 2011. Disponível em Acesso em 28 de dezembro de 2017.

Araújo Júnior, G. N., Silva, T. G. F., Souza, L. S. B., Souza, M. S., Araújo, G. G. L., Moura, M. S. B., Santos, J. P. A. S., Jardim, A. M. R. F., Alves, C. P., \& Alves, H. K. M. N. (2021). Productivity, bromatological composition and economic benefits of using irrigation in the forage cactus under regulated deficit irrigation in a semiarid environment. Bragantia, 80, e1221. https://doi.org/10.1590/1678-4499.20200390

Araújo, E. N. D., Oliveira, A. P. D., Cavalcante, L. F., Pereira, W. E., Brito, N. M. D., Neves, C. M. D. L., \& Silva, É. É. D. (2007). Produção do pimentão adubado com esterco bovino e biofertilizante. Revista brasileira de Engenharia Agrícola e Ambiental, 11(5), 466-470. http://dx.doi.org/10.1590/S141543662007000500003

Araújo, L. D. F., Oliveira, L. D. S., Perazzo Neto, A., de Alsina, O. L., \& da Silva, F. L. (2005). Equilíbrio higroscópico da palma forrageira: Relação com a umidade ótima para fermentação sólida. Revista Brasileira de Engenharia Agrícola e Ambiental, 9(3), 379-384. http://dx.doi.org/10.1590/S141543662005000300013

Attwood, G. T., Wakelin, S. A., Leahy, S. C., Rowe, S., Clarke, S., Chapman, D. F., ... \& Jacobs, J. M. (2019). Applications of the soil, plant and rumen microbiomes in pastoral agriculture. Frontiers in Nutrition, 6, 107. https://doi.org/10.3389/fnut.2019.00107

Barbosa, G., \& Langer, M. (2011). Uso de biodigestores em propriedades rurais: uma alternativa à sustentabilidade ambiental. Unoesc \& Ciência-ACSA, 2(1), 87-96.

Barbosa, M. L., Silva, T. G. F., Zolnier, S., Silva, S. M. S., Araújo Junior, G. N., \& Jardim, A. M. R. F. (2017). Meteorological variables and morphological characteristics influencing the evapotranspiration of forage cactus. Revista Ceres, 64(5), 465-475. https://doi.org/10.1590/0034-737x201764050003

Biesdorf, E. M., Jardim, A. M. R. F., Biesdorf, E. M., Barros, A. F., Araújo, C., Pimentel, L. D. (2019). Can the Nitrogen and Silicon Increase the Productivity and Yield in Rice Crops in the Rainfed Environment?. Journal of Agricultural Science, 11, 117-130. https://doi.org/10.5539/jas.v11n18p117

Boraste, A., Vamsi, K. K., Jhadav, A., Khairnar, Y., Gupta, N., Trivedi, S., ... \& Joshi, B. (2009). Biofertilizers: A novel tool for agriculture. International Journal of Microbiology Research, 1(2), 23-31.

Borges, F. R. M., Pinheiro Neto, L. G., Viana, T. V. A., Santos, A. P. G., Gomes do Ò, L. M., \& Lage, M. P. (2012). Crescimento e Desenvolvimento do Pimentão Híbrido Amarelo sob Energização da Água e Doses de Biofertilizante: Altura e Diâmetro Caulinar. Inovagri International Meeting. Fortaleza CE. p. $1-5$.

Borges, M., \& Bettiol, W. (2010). Agricultura Orgânica. EMBRAPA Meio Ambiente. Ministério da Agricultura e Abastecimento. 2p.

Borland, A. M., Hartwell, J., Weston, D. J., Schlauch, K. A., Tschaplinski, T. J., Tuskan, G. A., Yang, X., \& Cushman, J. C. (2014). Engineering crassulacean acid metabolism to improve water-use efficiency. Trends in Plant Science, 19, 327-338. http://dx.doi.org/10.1016/j.tplants.2014.01.006

Cândido, M. J. D., Gomes, G. M. F., Lopes, M. N., \& Ximenes, L. J. F. (2013). Cultivo da palma forrageira para mitigar a escassez de forragem em regiões semiáridas. Informe Rural, 7(3), 1-7. 
Cavalcanti, M. C. A., Batista, Â. M. V., Guim, A., Lira, M. A., Ribeiro, V. L., \& Ribeiro Neto, A. C. (2008). Consumo e comportamento ingestivo de caprinos e ovinos alimentados com palma gigante (Opuntia ficus-indica Mill) e palma orelha-de-elefante (Opuntia sp.). Acta Scientiarum. Animal Sciences, 30(2), 173179.

Carvalho, A. A., Silva, T. G. F., Queiroz, M. G., Araújo Júnior, G. N., Jardim, A. M. R. F., \& Souza, C. A. A. (2017). Estabilidade temporal e variabilidade espacial da umidade de um argissolo cultivado com palma forrageira. Revista Brasileira de Agricultura Irrigada, 11(7), 1998-2009. https://doi.org/10.7127/rbai.v11n700667

Chojnacka, K., Gorazda, K., Witek-Krowiak, A., \& Moustakas, K. (2019). Recovery of fertilizer nutrients from materials-Contradictions, mistakes and future trends. Renewable and Sustainable Energy Reviews, 110, 485-498. https://doi.org/10.1016/j.rser.2019.04.063

D’Andrea, P. A., \& Medeiros, M. B. (2002). Biofertilizantes biodinâmicos na nutrição e proteção de hortaliças. In: Congresso brasileiro de agricultura orgânica, natural, ecológica e biodinâmica, 1, Piracicaba. Anais... Piracicaba: Agroecológica.

Diniz Neto, M. A., Silva, I. D. F. D., Cavalcante, L. F., Diniz, B. L., da Silva, J. C., \& Silva, E. C. D. (2014). Mudas de oiticica irrigadas com águas salinas no solo com biofertilizante bovino e potássio. Revista Brasileira de Engenharia Agrícola e Ambiental, 18(1), 10-18. https://doi.org/10.1590/S141543662014000100002

Du Preez, C. C., Van Huyssteen, C. W., \& Mnkeni, P. N. (2011). Land use and soil organic matter in South Africa 2: A review on the influence of arable crop production. South African Journal of Science, 107(5-6), 35-42. http://dx.doi.org/10.4102/sajs.v107i5/6.358

Dubeux Júnior, J. C. B., \& Santos, M. V. F. (2005). Exigências nutricionais da palma forrageira. In: Menezes, R. S. C., Simões, D. A., \& Sampaio, E. V. S. B. (eds.). A palma no nordeste do Brasil: conhecimento atual e novas perspectivas de uso. 2.ed. Recife: Editora Universitária da UFPE.

Dubeux Júnior, J. C. B., Araújo Filho, J. T., Santos, M. V. F., Lira, M. A., Santos, D. C., \& Pessoa, R. A. S. (2013). Potential of cactus pear in South America. Cactusnet Newsletter, 13, ed. esp., 29-40.

EMBRAPA - Empresa Brasileira de Pesquisa Agropecuária (2010). Plantio e uso da palma forrageira na alimentação de bovinos leiteiros no semiárido brasileiro (Comunicado Técnico 62). Juiz de Fora, MG, Dezembro, 2010.

Farias, I., Santos, D. J., Dubeux Jr., J. C. B. (2005). Estabelecimento e manejo da palma forrageira. In: Menezes, R. S. C., Simões, D. A., Sampaio, E. V. S. B. (Ed.). A palma no Nordeste do Brasil: conhecimento atual e novas perspectivas de uso. Recife: Ed. Universitária da UFPE, 2005.

Fernandes, A. L. T., Rodrigues, G. P., \& Testezlaf, R. (2003). Mineral and organomineral fertirrigation in relation to quality of greenhouse cultivated melon. Scientia Agricola, 60(1), 149-154. https://doi.org/10.1590/S0103-90162003000100022

Figueroa, E. A., Escosteguy, P. A., \& Wiethölter, S. (2012). Dose de esterco de ave poedeira e suprimento de nitrogênio à cultura do trigo. Revista Brasileira de Engenharia Agrícola e Ambiental, 16(7), 714-720. https://doi.org/10.1590/S1415-43662012000700003

Galindo, I. C. L., Sampaio, E. V. S. B., \& Menezes, R. S. C. (2005). Uso da palma na conservação dos solos. A Palma No Nordeste Do Brasil: Conhecimento Atual e Novas Perspectivas de Uso. Menezes, R. S. C., Simões, D. A., Sampaio, E. V. S. B., Eds, 163-176.

Garcete-Gómez, J. M., Conforto, C., Domínguez-Monge, S., Flores-Sánchez, J. L., Mora-Aguilera, G., \& Michereff, S. J. (2017). Sample size for assessment of cladode brown spot in prickly pear cactus. European Journal of Plant Pathology, 149, (3), 1-5. https://doi.org/10.1007/s10658-017-1213-x

Gliessman, S. R. (2000). Agroecologia: processos ecológicos em agricultura sustentável. Trad. Maria José Guazzelli. Porto Alegre, UFRGS, 653p.

Hamoudi, S. A. A., \& Larich, A. (2013). Short-term effect of organic residue incorporation on soil aggregate stability along gradient in salinity in the lower cheliff plain (Algeria). African Journal of Agricultural Research, 8(19), 2141-2152.

Hrustek, L. (2020). Sustainability Driven by Agriculture through Digital Transformation. Sustainability, 12(20), 8596. https://doi.org/10.3390/su12208596

Izumi, M., Wada, S., Makino, A., \& Ishida, H. (2010). The autophagic degradation of chloroplasts via rubisco-containing bodies is specifically linked to leaf carbon status but not nitrogen status in Arabidopsis. Plant Physiology, 154(3), 1196-1209. https://doi.org/10.1104/pp.110.158519

Jardim, A. M. da R. F., Silva, T. G. F., Souza, L. S. B., Araújo Júnior, G. do N., Alves, H. K. M. N., Souza, M. de S., Araújo, G. G. L., \& Moura, M. S. B. (2021a). Intercropping forage cactus and sorghum in a semi-arid environment improves biological efficiency and competitive ability through interspecific complementarity. Journal of Arid Environments, 188. https://doi.org/10.1016/j.jaridenv.2021.104464

Jardim, A. M. R. F., Santos, H. R. B., Alves, H. K. M. N., Ferreira-Silva, S. L., Souza, L. S. B., Araújo Júnior, G. N., Souza, M. S., Araújo, G. G. L., Souza, C. A. A., \& Silva, T. G. F. (2021b). Genotypic differences relative photochemical activity, inorganic and organic solutes and yield performance in clones of the forage cactus under semi-arid environment. Plant Physiology and Biochemistry, 162, 421-430. https://doi.org/10.1016/j.plaphy.2021.03.011

Jardim, A. M. R. F., Silva, J. R. I., Leite, M. L. M. V., Teixeira, V. I., Morato, R. P., Araújo Júnior, G. N., \& Silva, T. G. F. (2018). Symbiotic interaction in forage crop cultivations: A review. Amazonian Journal of Plant Research, 2, 149-160. https://doi.org/10.26545/ajpr.2018.b00019x

Jardim, A. M. R. F., Silva, T. G. F., Souza, L. S. B., Alves, H. K. M. N., Araújo, J. F. N., Silva, G. I. N., \& Silva, J. O. N. (2019). Dinâmica da água no solo com cultivo de palma forrageira sob quatro sistemas de plantio. Agrometeoros, 27, 357-365. http://dx.doi.org/10.31062/agrom.v27i2.26446

Jardim, A. M. R. F., Silva, T. G. F., Souza, L. S. B., Souza, M. S., Morais, J. E. F., \& Araújo Júnior, G. N. (2020a). Multivariate analysis in the morpho-yield evaluation of forage cactus intercropped with sorghum. Revista Brasileira de Engenharia Agrícola e Ambiental, 24, 756-761. https://doi.org/10.1590/18071929/agriambi.v24n11p756-761

Jardim, A. M. R. F., Silva, T. G. F., Souza, L. S. B., \& Souza, M. S. (2020b). Interaction of agroecosystem intercropped with forage cactus-sorghum in the semi-arid environment: a review. Journal of Environmental Analysis and Progress, 5, 069-087. https://doi.org/10.24221/jeap.5.1.2020.2743.069-087 
Jardim, A. M. R. F., Silva, G. Í. N., Biesdorf, E. M., Pinheiro, A. G., Silva, M. V., Araújo Júnior, G. N., ... \& Silva, T. G. F. (2020c). Production potential of Sorghum bicolor (L.) Moench crop in the Brazilian semiarid. PUBVET, 14(4). https://doi.org/10.31533/pubvet.v14n4a550.1-13

Jardim, A. M. R. F., Araújo Júnior, G. D. N., Silva, M. J., Morais, J. E. F., \& Silva, T. G. F. (2017). Estimativas de perda de solo por erosão hídrica para o município de Serra Talhada, PE. Journal of Environmental Analysis and Progress, 186-193. https://doi.org/10.24221/jeap.2.3.2017.1416.186-193

Komiyama, T., Kobayashi, A., \& Yahagi, M. (2013). The chemical characteristics of ashes from cattle, swine and poultry manure. Journal of Material Cycles and Waste Management, 15(1), 106-110. https://doi.org/10.1007/s10163-012-0089-2

Kour, D., Rana, K. L., Yadav, A. N., Yadav, N., Kumar, M., Kumar, V., Vyas, P., Dhaliwal, H. S., \& Saxena, A. K. (2019). Microbial biofertilizers: Bioresources and eco-friendly technologies for agricultural and environmental sustainability. Biocatalysis and Agricultural Biotechnology, $23,101487$. https://doi.org/10.1016/j.bcab.2019.101487

Lana, R. P. (2009). Uso racional de recursos naturais não renováveis: aspectos biológicos, econômicos e ambientais. Revista Brasileira de Zootecnia, 38, 330340 .

Lassaletta, L., Billen, G., Grizzetti, B., Anglade, J., \& Garnier, J. (2014). 50 year trends in nitrogen use efficiency of world cropping systems: the relationship between yield and nitrogen input to cropland. Environmental Research Letters, 9(10), 105011. https://doi.org/10.1088/1748-9326/9/10/105011

Lima, L. R., Silva, T. G. F., Jardim, A. M. R. F., Souza, C. A. A., Queiroz, M. G., \& Tabosa, J. N. (2018). Growth, water use and efficiency of forage cactus sorghum intercropping under different water depths. Revista Brasileira de Engenharia Agrícola e Ambiental, 22(2), 113-118. https://doi.org/10.1590/18071929/agriambi.v22n2p113-118

Liu, M., Zhang, W., Wang, X., Wang, F., Dong, W., Hu, C., Liu, B., \& Sun, R. (2020). Nitrogen leaching greatly impacts bacterial community and denitrifiers abundance in subsoil under long-term fertilization. Agriculture, Ecosystems \& Environment, 294, 106885. https://doi.org/10.1016/j.agee.2020.106885

Lovatto, P. B., Watthier, M., Schiedeck, G., \& Schwengber, J. E. (2011). Efeito da urina de vaca como biofertilizante líquido na produção orgânica de mudas de couve (Brassica oleracea var. acephala). Horticultura Brasileira, 29, 4571-4577.

Maghanaki, M. M., Ghobadian, B., Najafi, G., \& Galogah, R. J. (2013). Potential of biogas production in Iran. Renewable and Sustainable Energy Reviews, 28, 702-714. https://doi.org/10.1016/j.rser.2013.08.021

Malavolta, E., Pimentel-Gomes, F., \& Alcarde, J. C. (2002). Adubos e adubações. São Paulo, Editora Nobel. 48p.

Marques, A. D. S., Paes, J. L., De Campos, D. V. B., Lima, E., Pinheiro, E. F. M., \& Matos, C. F. (2015). Efeito da aplicação do biofertilizante de dejetos de caprino no solo e cultura do sorgo. In: Congresso sobre geração distribuída e energia no meio rural, 10., 2015, São Paulo. Anais... São Paulo: Universidade de São Paulo.

Medeiros, D. C. D., Lima, B. A. B. D., Barbosa, M. R., Anjos, R. S. B. D., Borges, R. D., Cavalcante Neto, J. G., \& Marques, L. F. (2007). Produção de mudas de alface com biofertilizantes e substratos. Horticultura Brasileira, 25(3), 433-436. https://doi.org/10.1590/S0102-05362007000300021

Medeiros, R., Santos, B. J. M., Freitas, M., Silva, O. A., Alves, F. F., \& Ferreira, E. (2008). A adição de diferentes produtos químicos e o efeito da umidade na volatilização de amônia em cama de frango. Ciência Rural, 38(8), 2321-2326. https://doi.org/10.1590/S0103-84782008000800035

Meirelles, L., Bracagioli-Neto, A., Meirelles, A. L., Gonçalves, A., Guazzelli, M. J., Volpato, C., \& Bellé, N. (1997). Biofertilizantes enriquecidos, caminho sadio para a nutrição de plantas. Ipê: Centro de nutrição de plantas. Agricultura Ecológica de Ipê, $26 \mathrm{p}$.

Melo, R. F. M. R., Luiza, L. T. L. B., Pereira, L. A. P. L., \& Anjos, J. B. A. J. (2009). Avaliação do uso de adubo orgânico nas culturas de milho e feijão caupi em barragem subterrânea. Cadernos de Agroecologia, 4(1).

Menezes, R. S. C., Simões, D. A., Sampaio, E. V. S. B. (2005). A palma no nordeste do Brasil - conhecimento atual e novas perspectivas de uso. Recife, PE: Ed. Universitária, UFPE, 258p.

Meza-Herrera, C. A., Santamaría-Estrada, C. E., Flores-Hernández, A., Cano-Villegas, O., la Peña, C. G. De, Macias-Cruz, U., Calderón-Leyva, G., ÁngelGarcía, O., Mellado, M., Carrillo-Moreno, D., \& Véliz-Deras, F. G. (2019). The Opuntia effect upon the out-of-season embryo implantation rate in goats: Corpus luteal number, corpus luteal diameter and serum progesterone concentrations. Livestock Science, 228, 201-206. https://doi.org/10.1016/j.livsci.2019.09.002

Mitter, E. K., Tosi, M., Obregón, D., Dunfield, K. E., \& Germida, J. J. (2021). Rethinking Crop Nutrition in Times of Modern Microbiology: Innovative Biofertilizer Technologies. Frontiers in Sustainable Food Systems, 5, 29. https://doi.org/10.3389/fsufs.2021.606815

Moraes, J. G. V. (2015). Biofertilizantes: identificação das barreiras regulatórias e propostas para viabilizar esse insumo agrícola. $81 \mathrm{f}$. Dissertação (Mestrado em Agronegócio) - Escola de Economia de São Paulo da Fundação Getúlio Vargas, São Paulo.

Nascimento, A. C. O., Mattos, C. W., Dubeux Jr., J. C. B. et al. (2002). Desempenho da palma forrageira (Opuntia fícus indica Mill) submetida a diferentes níveis de adubação em Sertânia-PE. In: Congresso de iniciação cientifica UFRPE. Recife. Anais... Recife: Universidade Federal Rural do Pernambuco.

Nunes, C. S. (2011). Usos e aplicações da palma forrageira como uma grande fonte de economia para o semiárido nordestino. Revista Verde, 6, 58-66.

Nunes, J. S. L., Silva, T. G. F., Souza, L. S. B., Jardim, A. M. R. F., Alves, H. K. M. N., Cruz Neto, J. F., ... \& Pinheiro, A. G. (2019). Morfogênese da palma forrageira sob modificação do ambiente de crescimento. Agrometeoros, 27(2). http://dx.doi.org/10.31062/agrom.v27i2.26449

Oliveira, A. N. P., Oliveira, A. P., Leonardo, F. D. A. P., Cruz, I. D. S., \& Silva, D. F. (2009). Yield of gherkin in response to doses of bovine manure. Horticultura Brasileira, 27, 100-102. 
Owamah, H. I., Dahunsi, S. O., Oranusi, U. S., \& Alfa, M. I. (2014). Fertilizer and sanitary quality of digestate biofertilizer from the co-digestion of food waste and human excreta. Waste Management, 34(4), 747-752. https://doi.org/10.1016/j.wasman.2014.01.017

Paolini, V., Petracchini, F., Segreto, M., Tomassetti, L., Naja, N., \& Cecinato, A. (2018). Environmental impact of biogas: A short review of current knowledge. Journal of Environmental Science and Health, Part A, 53(10), 899-906. https://doi.org/10.1080/10934529.2018.1459076

Parawira, W. (2004). Anaerobic treatment of agricultural residues and wastewater. Doctoral Dissertation-Lund University, Sweden, 59 p.

Paré, T., Dinel, H., Schnitzer, M., \& Dumontet, S. (1998). Transformations of carbon and nitrogen during composting of animal manure and shredded paper. Biology and Fertility of Soils, 26(3), 173-178. https://doi.org/10.1007/s003740050364

Paungfoo-Lonhienne, C., Redding, M., Pratt, C., \& Wang, W. (2019). Plant growth promoting rhizobacteria increase the efficiency of fertilisers while reducing nitrogen loss. Journal of Environmental Management, 233, 337-341. https://doi.org/10.1016/j.jenvman.2018.12.052

Primavesi, A. C., Primavesi, O., Corrêa, L. D. A., Cantarella, H., Silva, A. G. D., Freitas, A. R. D., \& Vivaldi, L. J. (2004). Adubação nitrogenada em capimcoastcross: efeitos na extração de nutrientes e recuperação aparente do nitrogênio. Revista Brasileira de Zootecnia, 33(1), 68-78. https://doi.org/10.1590/S1516-35982004000100010

Probst, R., de Quadros, S. A. F., Erpen, J. G., \& Vincenzi, M. L. (2009). Produção de mudas de espécies forrageiras no sistema hidropônico de leito flutuante (floating) com solução nutritiva à base de biofertilizante ou adubo solúvel. Acta Scientiarum. Animal Sciences, 31(4), 349-355.

Ramos, J. P. F., Leite, M. L. M. V., Oliveira Junior, S., Nascimento, J. P., \& Santos, E. M. (2011). Crescimento vegetativo de Opuntia ficus-indica em diferentes espaçamentos de plantio. Revista Caatinga, 24(3), 41-48.

Restrepo Rivera, J. (2014). Manual práctico. El A, B, C de la Agricultura Organica y Harina de Roca. 84 p.

Riva, C., Orzi, V., Carozzi, M., Acutis, M., Boccasile, G., Lonati, S., Tambone, F., D’Imporzano, G., \& Adani, F. (2016). Short-term experiments in using digestate products as substitutes for mineral (N) fertilizer: agronomic performance, odours, and ammonia emission impacts. Science of The Total Environment, 547, 206-214. https://doi.org/10.1016/j.scitotenv.2015.12.156

Rothé, M., Darnaudery, M., \& Thuriès, L. (2019). Organic fertilizers, green manures and mixtures of the two revealed their potential as substitutes for inorganic fertilizers used in pineapple cropping. Scientia Horticulturae, 257, 108691. https://doi.org/10.1016/j.scienta.2019.108691

Sales, A. T., Alves, A. Q., Ramos, J. P. F., Nascimento, J. D., \& Leite, M. L. M. V. (2014). Eficiência de utilização da adubação orgânica pela palma forrageira em função da densidade populacional. Revista Científica de Produção Animal, 14, 32-35. http://dx.doi.org/10.15528/2176-4158/rcpa.v14n1p32-35

Sampaio, E. V. S. B. (2005). Fisiologia da palma. In: Menezes, R. S. C.; Simões, D. A.; Sampaio, E. V. S. B. (Ed.). A palma no Nordeste do Brasil: conhecimento atual e novas perspectivas de uso. Recife: Ed. Universitária da UFPE.

Santos, D. D., Farias, I., Lira, M. D. A., Santos, M. D., Arruda, G. D., Coelho, R. S. B., ... \& Melo, J. D. (2006). Manejo e utilização da palma forrageira (Opuntia e Nopalea) em Pernambuco. Recife: IPA, 48.

Santos, D. D., Farias, I., Lira, M. D. A., Tavares Filho, J. J., Santos, M. D., \& Arruda, G. D. (1997). A palma forrageira (Opuntia ficus-indica Mill e Nopalea cochenillifera Salm-Dyck) em Pernambuco: cultivo e utilização. Recife: IPA.

Schumacher, M. V., Caldeira, M. V. W., Oliveira, E. R. V. D., \& Piroli, E. L. (2001). Influência do vermicomposto na produção de mudas de Eucalyptus grandis Hill ex Maiden. Ciência Florestal, 11(2), 121-130. http://dx.doi.org/10.5902/198050981660

Silva, M. V., Pandorfi, H., de Almeida, G. L. P., de Lima, R. P., dos Santos, A., Jardim, A. M. R. F., ... \& da Silva, D. C. (2021a). Spatio-temporal monitoring of soil and plant indicators under forage cactus cultivation by geoprocessing in Brazilian semi-arid region. Journal of South American Earth Sciences, 107, 103155. https://doi.org/10.1016/j.jsames.2021.103155

Silva, J. O. N., Araújo Júnior, G. N., Jardim, A. M. R. F., Alves, C. P., Pinheiro, A. G., Santos, J. P. A. S., Souza, L. S. B., \& Silva, T. G. F. (2021b). Cultivation of forage cactus genotypes under biosalin agriculture as an alternative to increase forage input from the Brazilian semiarid region: A review. Research, Society and Development, 10(5), e16510514773. http://dx.doi.org/10.33448/rsd-v10i5.14773

Silva, M. L. N., Oliveira, F. D. A., de Oliveira, M. K. T., Maia, P. D. M. E., da Silva, R. C. P., \& da Silva, O. M. D. P. (2013). Efeito de biofertilizante bovino aeróbico na cultura do feijão caupi. Agropecuária Científica no Semiárido, 9(1), 110-116. http://dx.doi.org/10.30969/acsa.v9i1.271

Silva, J. R. I., Jardim, A. M. R. F., de Souza, E. S., Barros Junior, G., Leite, M. L. M. V., Souza, R., \& Antonino, A. C. D. (2020a). Inter-relação de técnica de manejo de água e solo aplicadas a cultura do milheto: uma revisão. Research, Society and Development, 9(7), e569974503. https://doi.org/10.33448/rsdv9i7.4503

Silva, P. F., Matos, R. M., Dantas Neto, J., Borges, V. E., Galvão Sobrinho, T., Bonou, S. I., Azevedo, C. A. V., Lima, V. L. A., \& de Melo Júnior, A. P. (2020b). Water and nitrogen water use efficiency in forage palm irrigated with salt water in the Neossolo. Australian Journal of Crop Science, 14, 683-690. https://doi.org/10.21475/ajcs.20.14.04.p2404

Silva, G. I. N., Jardim, A. M. R. F., Alves, H. K. M. N., Souza, M. S., Souza, C. A. A., Araújo Júnior, G. N., ... \& Silva, T. G. F. (2020c). Cultivo de plantas forrageiras de apelo regional para o Semiárido brasileiro sob a perspectiva de técnicas agrometeorológicas para melhoria da resiliência: uma revisão. Research, Society and Development, 9(10), e1449108291-e1449108291. https://doi.org/10.33448/rsd-v9i10.8291

Silva, T. G. F., Araújo Primo, J. T., Siqueira e Silva, S. M., Moura, M. S., Santos, D. C., Silva, M. C., \& Araújo, J. E. M. (2014). Indicadores de eficiência do uso da água e de nutrientes de clones de palma forrageira em condições de sequeiro no Semiárido brasileiro. Bragantia, 73(2), 184-191. http://dx.doi.org/10.1590/brag.2014.017 
Research, Society and Development, v. 10, n. 6, e46110616024, 2021

(CC BY 4.0) | ISSN 2525-3409 | DOI: http://dx.doi.org/10.33448/rsd-v10i6.16024

Souza, J. A. R., Moreira, D. A., Ferreira, P. A., \& Matos, A. T. D. (2009). Avaliação de frutos de tomate de mesa produzidos com efluente do tratamento primário da água residuária da suinocultura. Engenharia na Agricultura, 18(3), 198-207.

Souza, L. S. B., Moura, M. S. B., Silva, T. G. F., Soares, J. M., Carmo, J. F. A., \& Brandão, E. O. (2008). Indicadores climáticos para o zoneamento agrícola da palma forrageira (Opuntia sp.). In: Jornada de iniciação científica da Embrapa semiárido, 3, 2008, Petrolina. Documentos 210. Anais. Petrolina: Embrapa Semi-Árido, p. 23-28.

Souza, M. S., Jardim, A. M. R. F., Araújo Júnior, G. N., Silva, J. R. I., Leite, M. L. M. V., Teixeira, V. I., \& Silva, T. G. F. (2018). Ciclagem de nutrientes em ecossistemas de pastagens tropicais. PUBVET, 12, 172. https://doi.org/10.22256/pubvet.v12n5a91.1-9

Souza, M. T., Silva, M. D., \& Carvalho, R. (2010). Revisão integrativa: o que é e como fazer. Einstein, 8(1), 102-106. https://doi.org/10.1590/s167945082010 rw1134

Taiz, L., \& Zeiger, E. (2004). Fisiologia Vegetal. $3^{\mathrm{a}}$ edição. Porto Alegre: Artmed. 719p.

Trani, P. E., Terra, M. M., Tecchio, M. A., Teixeira, L. A. J., \& Hanasiro, J. (2013). Adubação Orgânica de Hortaliças e Frutíferas. Campinas (SP). 16 p.

Urra, J., Alkorta, I., Mijangos, I., \& Garbisu, C. (2020). Commercial and farm fermented liquid organic amendments to improve soil quality and lettuce yield. Journal of Environmental Management, 264, 110422. https://doi.org/10.1016/j.jenvman.2020.110422

Vasconcelos, A. G. V. D., Lira, M. D. A., Cavalcanti, V. L. B., Santos, M. V. F. D., \& Willadino, L. (2009). Seleção de clones de palma forrageira resistentes à cochonilha-do-carmim (Dactylopius sp). Revista Brasileira de Zootecnia, 38(5), 827-831. https://doi.org/10.1590/S1516-35982009000500007

Villela Junior, L. V. E., Araújo, J. A. C., \& Factor, T. L. (2003). Comportamento do meloeiro em cultivo sem solo com a utilização de biofertilizante. Horticultura Brasileira, 21(2), 154-158. https://doi.org/10.1590/S0102-05362003000200006 